\title{
Pretreatment with butorphanol reduces myoclonus after etomidate
}

\author{
Xiaohong Zhao', Rui Bao'2, Jiali Zhu², Zheng Liu², Yan Meng ${ }^{2}$, Xiaohua Fan² and Jinbao Li $^{2 *}$ \\ *Correspondence: lijinbaoshanghai@163.com or fxh95007@126.com \\ 'Department of Anesthesiology, General Hospital of Jinan Military Area, Ji Nan, China. \\ Department of Anesthesiology and Intensive Care Medicine, Changhai Hospital, Second Military Medical University, Shanghai, \\ China.
}

\begin{abstract}
1.Etomidate is often used in the induction of general anesthesia with a very stable hemodynamic profile. Myoclonus is a common problem during induction of anesthesia with etomidate, which may be a problem in the non-fasting patient. It has been shown that the incidence of myoclonus is decreased with a variety of opioid agents. Butorphanol is a strong analgesic with both narcotic agonist and antagonistic properties. This study aims to determine the efficacy of butorphanol and fentanyl for prevention of etomidate-induced myoclonus.

2.One hundred-fifty ASA |-|| patients, undergoing elective surgery were randomly assigned into 3 groups of 50 each. Butorphanol group received butorphanol $2 \mathrm{mg}$, fentanyl group received fentanyl $100 \mu \mathrm{g}$, and placebo group received normal saline. Ninety seconds after pretreatment patients received etomidate $0.3 \mathrm{mg} \cdot \mathrm{kg}^{-1}$. Assessment of myoclonus with IV etomidate was done by using a four-point scale: $0=$ no myoclonus, $1=$ mild myoclonus, $2=$ moderate myoclonus, and $3=$ severe myoclonus during a 60 -second period after etomidate injection.

3.In the placebo group $41(82 \%)$ patients had myoclonus after etomidate injection as compared to $12(24 \%)$ and $2(4 \%)$ in the fentanyl and butorphanol groups, respectively $(p<0.001)$.

4.Butorphanol is an effective and safe drug to reduce the etomidate-induced myoclonus without significant effects.
\end{abstract}

Key words: Etomidate, myoclonus, butorphanol, fentanyl

\section{Introduction}

Etomidate is a hypnotic agent causing minimal histamine release and a very stable hemodynamic profile. However, pain on injection and myoclonus are the most common side effects of this drug [1]. Pain on injection has been abolished by the new fat emulsion of etomidate, but the new solvent has not reduced the incidence of myoclonus [2]. Myoclonus is a common problem during induction of anesthesia with etomidate; up to $80 \%$ of non-premedicated patients develop myoclonic movements, [3] which may be a problem in the non-fasting patient because of the risk of hypoventilation as well as theoretically regurgitation and aspiration $[4,5]$. In patients with an open globe injury, myoclonus after etomidate raises the risk of prolapse of vitreous material as a result of high intraocular pressure [6]. It has been shown that the incidence of myoclonus is decreased with a variety of opioid agents such as fentanyl, sufentanil, alfentanil and remifentanil [7-10] Butorphanol is a synthetic, strong analgesic with both narcotic agonist and antagonistic properties. Butorphanol is often be used as an intraoperative analgesic [11]. However, no published data are yet available about the effects of butorphanol on myoclonus after injection of etomidate.

We designed a randomized, double-blind, placebo-controlled study of the effects of pretreatment with butorphanol and fentanyl on the incidence and severity of myoclonus during anesthesia induction with etomidate.

\section{Methods}

Approval for the study was obtained from the hospital research ethics committee and written informed consent obtained from each patient participating in the study. We expected the incidence of myoclonus in the placebo group to be 0.7 . Power analyses showed, using an $\alpha=0.05$ and $\beta=0.95$, that for a reduction rate of 0.35 a minimum of 49 patients would have to be included. We decided to include 50 patients per group. Statistical analyses were performed with SPSS 12.0 (SPSS, Chicago, IL). The Chi-squared test and the analyses of variance were used to identify statistical significance. A $p$ value of less than 0.05 was considered statistically significant.

One hundred and fifty ASA |-|| patients scheduled for surgery under general anesthesia without neurologic diseases were included. Midazolam $7.5 \mathrm{mg}$ was given orally $1 \mathrm{~h}$ before induction of anesthesia. An intravenous line was inserted in the patients' antecubital fossa after arrival in the operating room, and isotonic saline solution was infused at a rate of 15 to $20 \mathrm{ml} \cdot \mathrm{kg}-1$ per hour. Heart rate, noninvasive arterial blood pressure and oxygen saturation were recorded every minute during the study period.

Patients were allocated in a randomized and double blinded fashion to one of three pretreatment groups ( $n=50$ in each group): butorphanol $2 \mathrm{mg}$, fentanyl $100 \mu \mathrm{g}$ or saline as placebo. The drugs were prepared in a black syringe $(2 \mathrm{ml})$ outside the operating room, by a person not involved in the 
Zhao et al. Journal of Anesthesiology and Clinical Science 2013,

Table 1. Patients' Characteristics. Values are mean(SD) or number.

\begin{tabular}{cccc}
\hline & Placebo & Fentanyl & Butorphanol \\
\hline Age;years & $50 \pm 10$ & $47 \pm 15$ & $48 \pm 13$ \\
Sex(m:f) & $25 / 25$ & $24 / 26$ & $23 / 27$ \\
ASA physical status & $28 / 22$ & $26 / 24$ & $24 / 26$ \\
|/I (n) & & & $56.7 \pm 13.8$ \\
Weight; $\mathrm{kg}$ & $55.5 \pm 10.2$ & $58.2 \pm 12.2$ & $169 \pm 10$ \\
Height; cm & $168 \pm 8$ & $166 \pm 6$ &
\end{tabular}

Table 2. The incidence and severity of myoclonus in all groups.

\begin{tabular}{cccccc}
\hline & Grade 0 & Grade 1 & Grade 2 & $\begin{array}{c}\text { Grade } \\
\mathbf{3}\end{array}$ & $\begin{array}{c}\text { Total My- } \\
\text { oclonus }\end{array}$ \\
\hline $\begin{array}{c}\text { Placebo } \\
\text { group(n=50) }\end{array}$ & 9 & 7 & 18 & 16 & 41 \\
$\begin{array}{c}\text { Fentanyl } \\
\text { groupw(n=50) } \\
\text { Butorphanol } \\
\text { group(n=50) }\end{array}$ & $38^{*}$ & 4 & 6 & 2 & $12^{*}$ \\
\hline
\end{tabular}

${ }^{*} \mathrm{p}<0.001$ vs Placebo group. \# $\mathrm{p}<0.001$ vs Fentanyl group.

induction of anesthesia. Randomization was achieved using a computer-generated list. The anaesthetist performing the induction and the patient were not aware of the pretreatment drug. The patients were pre-oxygenated and the study drug was infused. After a ninety-second interval, etomidate 0.3 mg.kg-1 (Etomidate-LipuroR, B. Braun Melsungen, Melsungen Germany) was injected intravenously over 30 seconds. After injection of the test drug, the grade of sedation (none, mild, moderate, severe), nausea, and apnea were recorded. Patients were observed visually for myoclonus, and when present, myoclonus severity was graded one minute after the end of the etomidate injection by a trained physician who was blinded to the treatment groups. Myoclonus was defined as involuntary, short contraction of some muscle fibers, of a whole muscle, or of different muscles of one group, leading to short observable movements of the corresponding body parts. The intensity of myoclonic movement was graded as $0=$ no myoclonus, $1=$ mild myoclonus, $2=$ moderate myoclonus, and 3 = severe myoclonus [3]. One minute after etomidate administration and evolution of myoclonus, rocuronium $0.6 \mathrm{mg} \cdot \mathrm{kg}-1$ was given to facilitate tracheal intubation. At that point, fentanyl $100 \mu \mathrm{g}$ was given in the placebo group to guarantee adequate anesthesia. No other drugs were used between premedication and the end of induction. The trachea was intubated and anesthesia was maintained with isoflurane or sevoflurane in oxygen.

\section{Results}

There were no significant differences among the groups in the patients' demographic data (Table 1).

Only two of $50(4 \%)$ of the patients pretreated with butorphanol, but 41 of $50(82 \%)$ of the patients with placebo, and 12 of 50 (24\%) of the patients with fentanyl, developed myoclonus during the 60 -second period following induction with etomidate $(p<0.001)$ (Table 2 and Figure 1$)$. The intensity of myoclonus is also shown in Table 2 and Figure 1.

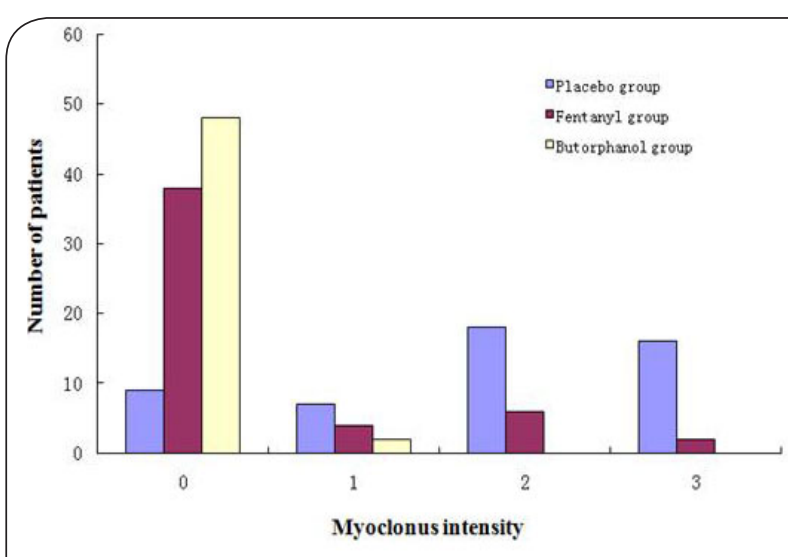

Figure 1. Assessment of myoclonus.

Number of adult patients who experienced myoclonus during the onemin observation period after an etomidate injection in each treatment group. Placebo group received saline pre-treatment $(n=50)$; Fentanyl group received fentanyl $1 \mu \mathrm{g} / \mathrm{kg}$ pretreatment $(\mathrm{n}=50)$; and Butorphanol group received Butorphanol $2 \mathrm{mg} / \mathrm{kg}$ pre-treatment $(\mathrm{n}=50) . \mathrm{P}<0.001$ in the incidence of myoclonus for fentanyl group and butorphanol group versus placebo group; Butorphanol group versus fentanyl group, significantly different for the severity of myoclonus.

Between pretreatment and induction of anesthesia with etomidate all patients in all pretreatment groups remained conscious. The three groups were comparable with regard to heart rate and blood pressure values during the study period. None of our patients experienced apnea, nausea, bradycardia, or hypotension after injection of pretreatment drugs.

\section{Discussion}

We observed that pretreatment with butorphanol $2 \mathrm{mg}$ or fentanyl $100 \mu \mathrm{g}$ reduced the incidence and severity of myoclonus associated with IV injection of etomidate $(p<0.001)$. Butorphanol pretreatment was most effective in attenuating the incidence and severity of myoclonus associated with IV injection of etomidate $(p<0.001)$.

Sung et al., found that the butorphanol was a good opioid analgesic for balanced anaesthesia [12]. The authors suggested that butorphanol was a better choice than morphine for use in balanced anaesthesia techniques because of its comparable analgesic efficacy and amnesia along with lesser postoperative respiratory depression and a shorter recovery room stay. We chose the dose of butorphanol $2 \mathrm{mg}$ because it has been shown to be effective in the prevention of pain during the injection of propofol [13]. The analgesia effect of butorphanol $2 \mathrm{mg}$ was similar to that of fentanyl 100 $\mathrm{\mu g}$ [14]. So, in this study we chose the fentanyl dose $(100 \mu \mathrm{g})$ that was previously used to prevent myoclonus during etomidate induction [7].

The neurologic mechanism of myoclonus after etomidate is unclear. Kugler et al ., [15]. postulated that inhibitory circuits can be depressed earlier and at lower etomidate concentrations than excitatory neuronal circuits. Doenicke et al., 
observed that myoclonus after etomidate is a phenomenon of subcortical disinhibition like the phenomenon of restless legs during normal human sleep and is not generated by an epileptic focus [3].

Various methods have been used for attenuating myoclonus during IV injection of etomidate. Pretreatment with diazepam or flunitrazepam failed to reduce myoclonus, [16 ]but midazolam reduced the incidence because of faster onset [17]. Doenicke et al., reported that pretreatment with three different dosages(etomidate $0.03,0.05$ or $0.075 \mathrm{mg} / \mathrm{kg}$ IV) of etomidate reduced myoclonus in a trial $(n=20)$ without a control group in premedicated patients [3]. Pretreatment with fentanyl $100 \mu \mathrm{g}$ reduced the incidence of myoclonus to $8 \%$ [18]. The use of larger fentanyl dosages, however, increased the incidence of apnea during induction [ 7]. The incidence of myoclonus after pretreatment with alfentanil $5 \mu \mathrm{g} \cdot \mathrm{kg}-1$ was reported to be $25 \%$ [8]. Hueter et al., examined pretreatment with sufentanil $0.3 \mu \mathrm{g} \cdot \mathrm{kg}-1$ on the incidence and severity of myoclonus after etomidate in female patients [7]. Myoclonus was observed in none of the patients in the sufentanil group, but in $80 \%$ of patients in the placebo group. Sixty percent of the patients treated with sufentanil showed some degree of sedation from mild to severe, whereas only $15 \%$ in the placebo group. Although it was shown that fentanyl, alfentanil, or sufentanil are effective in reducing myoclonus, these agents are more suitable for longer surgical procedures. During short or outpatient procedures, these agents may cause residual apnea, sedation, nausea, vomiting, and delayed discharge from the hospital. Another opioid with very short onset time, remifentanil $\left(1 \mathrm{\mu g}^{\prime} \mathrm{kg}^{-1}\right)$, was very effective in reducing myoclonus after etomidate from $70 \%$ in the placebo group to $6.7 \%$ in the remifentanil group [10]. However, remifentanil can cause severe bradycardia. Klausen showed that buprenorphine has no influence on the incidence of myoclonus [19]. Guler et al., reported that $\mathrm{Mg} 2.48 \mathrm{mmol}$ administered $90 \mathrm{~s}$ before the induction of anesthesia with etomidate was effective in reducing the incidence and severity of etomidate-induced myoclonus and that ketamine did not reduce the incidence of myoclonus [20].

Butorphanol tartrate is a mixed agonist-antagonist with intrinsic activity at receptors of the mu opioid type (morphine like). It is also an agonist at kappa opioid receptors. Its interactions with these receptors in the CNS apparently mediate most of its pharmacological effects, including analgesia. Butorphanol has an analgesic action 5-8 times more potent than morphine, with less respiratory depression, less nausea and vomiting, no undesirable psychomimetic effects and the provision of perioperative amnesia. After IV administration the onset of analgesia occurs rapidly (within 1-2 minute) with peak effect occurring in about 4-5 minutes [21]. The site of action of butorphanol in reducing the myoclonus of etomidate injection is not clear. Manocha et al., examined the effect of butorphanol on convulsive behaviour using maximal electroshock (MES) test [22].Their results implicated a role for multitude of neurotransmitter systems, i.e., opioid (mu, kappa, delta), NMDA channel, BZD-GABAA chloride channel complex, and GABAB receptors in the anti-MES action of butorphanol. The analgesic action and the sedative effect of butorphanol may contribute to reducing of myoclonus after etomidate administration. Further work is suggested for confirming the mechanism of action of butorphanol in preventing etomidate induced myoclonus.

We used midazolam, a short-acting benzodiazepine, in all patients as premedication one hour before induction of anesthesia for ethical reasons. In spite of midazolam premedication, the incidence of myoclonus was high in the placebo group. Butorphanol has excellent sedative effect alone and especially in combination with other agents such as promethazine [23]. The combination of butorphanol and etomidate may lead to profound sedation, but prolong awakening was not observed in all of our patients.

We conclude that pretreatment with butorphanol $2 \mathrm{mg}$ before induction with $0.3 \mathrm{mg} \cdot \mathrm{kg}-1$ etomidate reduces the incidence of myoclonus without any side effects such as sedation, apnea, nausea, or bradycardia. Butorphanol can be a good choice for prevention of myoclonus after etomidate administration. However, it is believed that independent studies will prove further demonstration of its efficacy and tolerability.

\section{Competing interests}

The authors declare that they have no competing interests.

\section{Authors' contributions}

XZ and RB: planned study, analyzed data and drafted the manuscript. They contributed equally to the manuscript; JZ: collected and analyzed data;

ZL: collected data;

YM: collected and analyzed data;

XF and JL: planned study, drafted and revised the manuscript. They contributed equally to the manuscript.

Publication history

Received: 21-Nov-2012 Revised: 10-Dec-2012

Accepted: 20-Dec-2012 Published: 11-Jan-2013

\section{References}

1. A Doenicke, MF Roizen, AE Nebauer, A Kugler, R Hoernecke and $\mathrm{H}$ Beger-Hintzen: A comparison of two formulations for etomidate, 2-hydroxypropyl-beta-cyclodextrin (HPCD) and propylene glycol. Anesth Analg 1994, 79:933-9. I PubMed

2. JM Bergen and DC Smith: A review of etomidate for rapid sequence intubation in the emergency department. J Emerg Med 1997, 15:22130. | Article | PubMed |

3. AW Doenicke, MF Roizen, J Kugler, H Kroll, J Foss and P Ostwald: Reducing myoclonus after etomidate. Anesthesiology 1999, 90:113-9. I PubMed

4. SG Van Keulen and JH Burton: Myoclonus associated with etomidate for ED procedural sedation and analgesia. Am J Emerg Med 2003, 21:556-8. | Article | PubMed

5. L Huter, T Schreiber, M Gugel and K Schwarzkopf: Low-dose intravenous midazolam reduces etomidate-induced myoclonus: a prospective, randomized study in patients undergoing elective cardioversion. Anesth 
Zhao et al. Journal of Anesthesiology and Clinical Science 2013,

http://www.hoajonline.com/journals/pdf/2049-9752-2-2.pdf

Analg 2007, 105:1298-302. | Article | PubMed

6. JM Berry and RG Merin: Etomidate myoclonus and the open globe. Anesth Analg 1989, 69:256-9. | PubMed

7. RJ Stockham, TH Stanley, NL Pace, S Gillmor, F Groen and P Hilkens: Fentanyl pretreatment modifies anaesthetic induction with etomidate. Anaesth Intensive Care 1988, 16:171-6. | PubMed

8. L Hueter, K Schwarzkopf, M Simon, D Bredle and H Fritz: Pretreatment with sufentanil reduces myoclonus after etomidate. Acta Anaesthesiol Scand 2003, 47:482-4. | Article | PubMed

9. SN Khalil, KS Lawson, CL Hanis, NA Lemak and RS Ruiz: Alfentanil decreases myoclonus caused by etomidate. Middle East J Anesthesiol 1999, 15:185-92. | PubMed

10. E Kelsaka, D Karakaya, B Sarihasan and S Baris: Remifentanil pretreatment reduces myoclonus after etomidate. J Clin Anesth 2006, 18:83-6. | Article | PubMed

11. L Mishra, N Rajkumar, S Singh, R Dubey and G Yadav: A Comparative Study of Propofol and Isoflurane Anaesthesia using Butorphanol in Neurosurgery. Indian J Anaesth 2009, 53:324-9. | PubMed Abstract | PubMed Full Text

12. YF Sung, MS Weinstein and GA Ghani: Balanced anesthesia: a comparison of butorphanol and morphine. South Med J 1984, 77:1802. | PubMed

13. A Agarwal, M Raza, S Dhiraaj, R Pandey, D Gupta, CK Pandey, PK Singh and U Singh: Pain during injection of propofol: the effect of prior administration of butorphanol. Anesth Analg 2004, 99:117-9. | Article I PubMed

14. Jaffe JH, Martin WR. Opioid analgesics and antagonists. In: Gilman AG, Goodman LS, Gilman A (eds.), Pharmacological basis of therapeutics. $8^{\text {th }}$ ed, New York: Macmillan, 1990;508-513.

15. H Suttmann, A Doenicke, J Kugler and M Laub: [A new formulation of etomidate in lipid emulsion--bioavailability and venous provocation]. Anaesthesist 1989, 38:421-3. | PubMed

16. K Korttila, T Tammisto and U Aromaa: Comparison of etomidate in combination with fentanyl or diazepam, with thiopentone as an induction agent for general anaesthesia. Br J Anaesth 1979, 51:1151-7. | Article | PubMed

17. KR Schwarzkopf, L Hueter, M Simon and HG Fritz: Midazolam pretreatment reduces etomidate-induced myoclonic movements. Anaesth Intensive Care 2003, 31:18-20. e I PubMed

18. JL Giese, RJ Stockham, TH Stanley, NL Pace and RH Nelissen: Etomidate versus thiopental for induction of anesthesia. Anesth Analg 1985, 64:871-6. | PubMed

19. N Klausen, SH Johansen, F Janstrup and JG Hansen: Preoperative buprenorphine does not prevent myoclonia seen after etomidate. $\mathrm{Br} J$ Anaesth 1982, 54:475. | Article | PubMed

20. A Guler, T Satilmis, SB Akinci, B Celebioglu and M Kanbak: Magnesium sulfate pretreatment reduces myoclonus after etomidate. Anesth Analg 2005, 101:705-9. | Article | PubMed

21. Lippmann M, Mok MS, Steen SN: Analgesic onset time of intravenous butorphanol in postsurgical patients: a placebo-controlled study. Curr Ther Res 1977, 22:276-83.

22. A Manocha, KK Sharma and PK Mediratta: Possible mechanism involved in the anticonvulsant action of butorphanol in mice. Pharmacol Biochem Behav 2003, 74:343-50. | Article | PubMed

23. M Lippmann, MS Mok and SN Steen: Butorphanol and promethazine as pre-anaesthetic medication. J Int Med Res 1978, 6:455-9. | PubMed

\section{Citation:}

Zhao X, Bao R, Zhu J, Liu Z, Meng Y, Fan X and Li J:

Pretreatment with butorphanol reduces myoclonus after etomidate. journal of Anesthesiology and Clinical Science

2013, 2:2. http://dx.doi.org/10.7243/2049-9752-2-2 